\title{
Construction of integrable models on ladders, and related quantum symmetries
}

\section{Arnaudon ${ }^{* \dagger}$}

Laboratoire d'Annecy-Le-Vieux de Physique Théorique LAPTH ${ }^{\ddagger}$

B.P. 110, F-74941 Annecy-Le-Vieux cedex, France

E-mail: arnaudon@lapp. in2p3. fr

AbstraCt: A spin chain based on the XXZ model is constructed, with staggered disposition of the spectral parameter and of the anisotropy parameter. Its integrability is proved and the corresponding Hamiltonian is derived. It can be interpreted as a ladder Hamiltonian. The symmetry of the model is an extension of the $\mathcal{U}_{q}(s l(2))$ algebra.

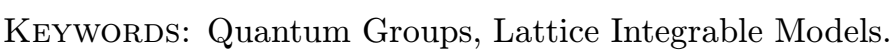

This talk is based on works done with R. Poghossian, A. Sedrakyan, T. Sedrakyan and P. Sorba. More details may be found in [i], as well as relevant references. The last part is still in progress.

We will present a spin chain model based on the XXZ chain. It will provide a Hamiltonian with next-to-rearest neighbour interaction. In the free fermionic limit, this model decouples into two (even and odd) sub-chains, indicating that a ladder chain interpretation of the Hamiltonian is physically interesting. The investigation of the possible generalisations of this model to more complicated ladder may have interesting applications in the theory of superconductivity (see the report of Alessandro De Martino [2] on the physics of ladder chains).

\section{Description of the model: stag- gered monodromy matrix}

We start from the XXZ $\mathcal{R}$-matrix and use it as a building block of a staggered model. We consider $\mathbb{Z}_{2}$ graded auxiliary and quantum spaces $V_{a, \sigma}(u)$

\footnotetext{
${ }^{*}$ Work done with R. Poghossian, A. Sedrakyan, T. Sedrakyan and P. Sorba.

†Work partially supported by European Community contract TMR FMRX-CT96.0012

‡UMR 5108 du CNRS, associée à l'Université de Savoie
}

and $V_{j, \rho}(v),(\sigma, \rho=0,1)$ (without fermionic interpretation), and consider four different R-matrices

$$
\begin{aligned}
& R_{a j, \sigma \rho}(u, v): \\
& \quad V_{a, \sigma}(u) \otimes V_{j, \rho}(v) \rightarrow V_{j, \bar{\rho}}(v) \otimes V_{a, \bar{\sigma}}(u),
\end{aligned}
$$

with $\bar{\sigma}=(1-\sigma), \bar{\rho}=(1-\rho)$.

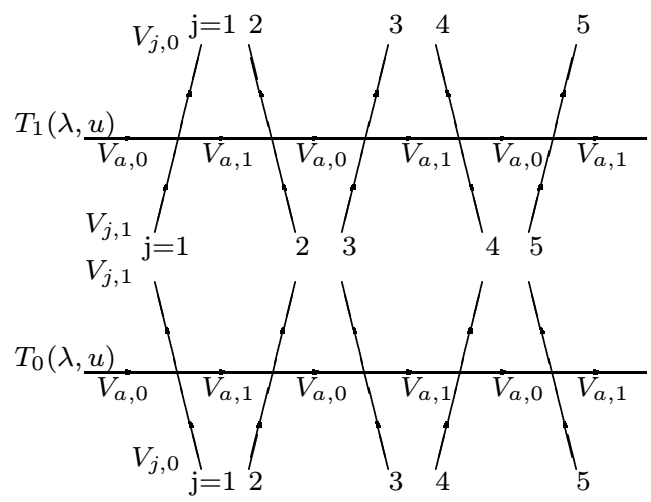

Figure 1: Monodromy matrices $T_{0}$ and $T_{1}$

It is convenient to introduce two "transmutation" operations $\iota_{1}$ and $\iota_{2}$ with the property $\iota_{1}^{2}=\iota_{2}^{2}=i d$ for the quantum and auxiliary spaces correspondingly, and to mark the operators $R_{a j, \sigma \rho}$ as follows

$$
R_{a j, 00} \equiv R_{a j}, \quad R_{a j, 01} \equiv R_{a j}^{\iota_{1}},
$$




$$
R_{a j, 10} \equiv R_{a j}^{\iota_{2}}, \quad R_{a j, 11} \equiv R_{a j}^{\iota_{1} \iota_{2}}
$$

We then introduce a double line monodromy matrix as

$$
T(\lambda, u)=T_{0}(\lambda, u) T_{1}(\lambda, u)
$$

where $T_{0}$ and $T_{1}$ are staggered products of $\iota_{2}$ transmuted $R$-matrices

$$
\begin{aligned}
& T_{1}(\lambda, u)=\prod_{j=1}^{N} R_{a, 2 j-1}(\lambda, u) \bar{R}_{a, 2 j}^{\iota_{2}}(\lambda, u) \\
& T_{0}(\lambda, u)=\prod_{j=1}^{N} \bar{R}_{a, 2 j-1}^{\iota_{1}}(\lambda, u) R_{a, 2 j}^{\iota_{1} \iota_{2}}(\lambda, u),
\end{aligned}
$$

and where the notation $\bar{R}$ in general means the different parameterization of the $R$-matrix via model $\lambda$ and spectral $u$ parameters and can be considered as an operation over $R$ with property $\overline{\bar{R}}=R$.

In order to have a integrable model with commuting transfer matrices (11.3) for different spectral parameters

$$
[\operatorname{tr} T(\lambda, u), \operatorname{tr} T(\lambda, v)]=0
$$

it is enough to have the following relations for the $\tau_{\sigma}(u)=\operatorname{tr} T_{\sigma}(\lambda, u), \quad(\sigma=0,1)$

$$
\tau_{\sigma}(\lambda, u) \tau_{1-\sigma}(\lambda, v)=\bar{\tau}_{\sigma}(\lambda, v) \bar{\tau}_{1-\sigma}(\lambda, u),
$$

which is true if the two local condition (YangBaxter like equations) are satisfied

$$
\begin{aligned}
& R_{12}(u, v) \bar{R}_{13}^{\iota_{1}}(u) R_{23}(v)= \\
& R_{23}^{\iota_{1}}(v) \bar{R}_{13}(u) \tilde{R}_{12}(u, v)
\end{aligned}
$$

and

$$
\begin{aligned}
& \tilde{R}_{12}(u, v) R_{13}^{\iota_{1} \iota_{2}}(u) \bar{R}_{23}^{\iota_{2}}(v)= \\
& \bar{R}_{23}^{\iota_{1} \iota_{2}}(v) R_{13}^{\iota_{2}}(u) R_{12}(u, v),
\end{aligned}
$$

for some $\tilde{R}_{12}$.

A solution of (11.11) is given by

$$
\begin{aligned}
& R(u)= \\
& \left(\begin{array}{cccc}
\sin (\lambda+u) & 0 & 0 & 0 \\
0 & \sin (u) & e^{-i u} \sin (\lambda) & 0 \\
0 & e^{i u} \sin (\lambda) & \sin (u) & 0 \\
0 & 0 & 0 & \sin (\lambda+u)
\end{array}\right),
\end{aligned}
$$

$$
\begin{aligned}
& R^{\iota_{1}}(u)= \\
& \left(\begin{array}{cccc}
\sin (\lambda+u) & 0 & 0 & 0 \\
0 & -\sin (u) & e^{-i u} \sin (\lambda) & 0 \\
0 & e^{i u} \sin (\lambda) & -\sin (u) & 0 \\
0 & 0 & 0 & \sin (\lambda+u)
\end{array}\right),
\end{aligned}
$$

with furthermore

$$
\begin{aligned}
R(u, v) & \equiv R(\theta-u-v), \\
\bar{R}(u) & \equiv R(\theta-u), \\
R^{\iota_{2}}(u) & =R^{\iota_{1}}(-u), \\
\tilde{R}_{12}(u) & =R^{\iota_{1}}(u) .
\end{aligned}
$$

(Notice that we introduced here the off-diagonal factors $e^{i u}$ and $e^{-i u}$ not present in [i] to allow the later decomposition (4.1). They are nothing more than a rescaling of the states or a simple gauge transformation.)

Writing $R$ as

$$
\begin{aligned}
R_{j k}(u) & =a(u)\left[-n_{j} n_{k}+\left(1-n_{j}\right)\left(1-n_{k}\right)\right] \\
& +b(u)\left[n_{j}\left(1-n_{k}\right)+\left(1-n_{j}\right) n_{k}\right] \\
& +\xi c(u) c_{j}^{+} c_{k}+\xi^{-1} c(u) c_{k}^{+} c_{j},
\end{aligned}
$$

$\Delta$, the anisotropy parameter of the XXZ model, is defined by

$$
\Delta=\frac{a^{2}(u)+b^{2}(u)-c^{2}(u)}{2 a(u) b(u)}
$$

actually does not depend on the spectral parameter.

It is important to mention here that the transmutations $\iota_{1}$ and $\iota_{2}$ change the sign of $\Delta$, as

$$
\Delta_{\iota_{1}}=\Delta_{\iota_{2}}=-\Delta
$$

This means, that in the definition (11.4) of the monodromy matrices $T_{0,1}(\lambda, u)$ we have a product of $R$-matrices with alternating anisotropy parameters $\pm \Delta$.

\section{The Hamiltonian}

The derivation of a Hamiltonian is usually done using

$$
H=-\left.\frac{\partial \ln \operatorname{tr} T(u)}{\partial u}\right|_{u=u_{0}} .
$$


The locality of the Hamiltonian comes from the fact that, for some special value of the spectral parameter $u_{0}$, one has

$$
\begin{array}{lc} 
& \check{R}_{i j}\left(u_{0}\right)=I_{i j} \\
\text { with } & \check{R}=P R .
\end{array}
$$

In our case, not all the $\check{R}$-matrices of the monodromy matrix are identity for $u=u_{0}$. We nevertheless have a local Hamiltonian obtained as

$$
\begin{aligned}
T(u) \approx\left(\sin ^{2} \lambda\right. & \sin (\lambda+\theta) \sin (\lambda-\theta))^{N} . \\
& \left(1+u \sum_{j=1}^{N} H_{j}\right),
\end{aligned}
$$

where $H$ contains (local) interaction of the form

$$
\begin{aligned}
H_{j}= & h_{1}\left(1-n_{j-1}-n_{j+2}\right)\left(c_{j}^{+} c_{j+1}-c_{j+1}^{+} c_{j}\right) \\
& +h_{2}\left[n_{j} n_{j+2}+\left(1-n_{j}\right)\left(1-n_{j+2}\right)\right] \\
& +\left(h_{3}\left(1-n_{j+1}\right)+h_{4} n_{j+1}\right) c_{j}^{+} c_{j+2} \\
& -\left(h_{4}\left(1-n_{j+1}\right)+h_{3} n_{j+1}\right) c_{j+2}^{+} c_{j} \cdot(2.5)
\end{aligned}
$$

In the $\Delta=0$ limit,

$$
H_{j}=\left[c_{j}^{+} c_{j+2}-c_{j+2}^{+} c_{j}\right] .
$$

which corresponds to free fermions, propagating separately on even and odd sub lattices.

It is then convenient to write the Hamiltonian $(2.5)$ in a ladder form represented graphically as in Figure 2.

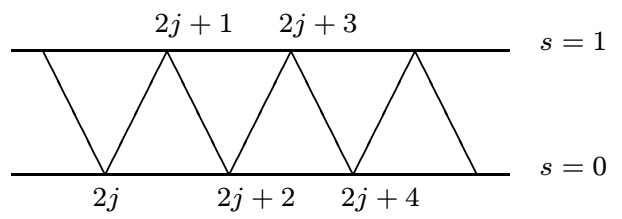

Figure 2: Zig-zag ladder chain

Defining indeed

$$
c_{j, s}=c_{2 j+s}, \quad s=0,1
$$

where $s$ labels the legs of the ladder, we get

$$
\begin{aligned}
& H_{j, s}= \\
& h_{1}\left(c_{j, s}^{+} c_{j, s+1}-c_{j, s+1}^{+} c_{j, s}\right)\left(\bar{n}_{j-1, s+1}-n_{j+1, s}\right) \\
& +h_{2}\left[n_{j, s} n_{j+1, s}+\left(1-n_{j, s}\right)\left(1-n_{j+1, s}\right)\right] \\
& +c_{j, s}^{+} c_{j+1, s}\left(h_{3}\left(1-n_{j, s+1}\right)+h_{4} n_{j, s+1}\right) \\
& -c_{j+1, s}^{+} c_{j, s}\left(h_{3} n_{j, s+1}+h_{4}\left(1-n_{j, s+1}\right)\right) .
\end{aligned}
$$

This Hamiltonian contains hopping terms along each leg of the ladder (depending on the occupation number on the other rung), and also hopping term from one rung to the other.

\section{Bethe Ansatz equations}

The monodromy matrices $T_{0}$ and $T_{1}$ act as (the blocks corresponding to auxiliary space indices)

$$
\begin{aligned}
\left(T_{s}(u)\right)_{a}^{a^{\prime}}= & \left\langle a^{\prime}\left|T_{s}(u)\right| a\right\rangle \\
= & \left(\begin{array}{cc}
A_{s}(u) & B_{s}(u) \\
C_{s}(u) & D_{s}(u)
\end{array}\right), \\
& (s=0,1)
\end{aligned}
$$

The reference state

$$
|\Omega\rangle=\prod_{i=1}^{2 N}|0\rangle_{i}
$$

is an eigenstate of $T_{0} T_{1}$.

Other eigenstates are as usual obtained as

$$
|V\rangle_{0}=B_{1}\left(v_{1}\right) B_{0}\left(v_{2}\right) \ldots B_{x}\left(v_{n}\right)|\Omega\rangle
$$

for particular values of the parameters $v_{i}$, that we will now characterise.

From the RTT relations satisfied by the monodromy matrices, one deduces

$$
\begin{aligned}
A_{0}(u) B_{1}(v)= & -\frac{a(v-u)}{b(v-u)} B_{0}(v) A_{1}(u) \\
& +\frac{c(v-u)}{b(v-u)} B_{0}(u) A_{1}(v) \\
A_{1}(u) B_{0}(v)= & +\frac{a(v-u)}{b(v-u)} B_{1}(v) A_{0}(u) \\
& -\frac{c(v-u)}{b(v-u)} B_{1}(u) A_{0}(v)
\end{aligned}
$$

and

$$
\begin{aligned}
D_{0}(u) B_{1}(v)= & +\frac{a(u-v)}{b(u-v)} B_{0}(v) D_{1}(u) \\
& +\frac{c(u-v)}{b(u-v)} B_{0}(u) D_{1}(v) \\
D_{1}(u) B_{0}(v)= & -\frac{a(u-v)}{b(u-v)} B_{1}(v) D_{0}(u) \\
& -\frac{c(u-v)}{b(u-v)} B_{1}(u) D_{0}(v) .
\end{aligned}
$$

Demanding that, in the action of the monodromy matrix on $\left(3.3^{i}\right)$, no term of the form $B(u) X\left(v_{i}\right)$ 
for $X=A, D$ is left (so-called "unwanted term"), we get the Bethe equations for the $v_{i}$ :

$$
\begin{gathered}
{\left[\frac{a\left(v_{j}\right) a^{\iota_{2}}\left(\bar{v}_{j}\right)}{b\left(v_{j}\right) b^{\iota_{2}}\left(\bar{v}_{j}\right)}\right]^{N}=-\prod_{i \neq j}^{n} \frac{a\left(v_{j}-v_{i}\right)}{a\left(v_{i}-v_{j}\right)},} \\
j=1, \ldots, n .
\end{gathered}
$$

\section{Reverse Baxterisation procedure}

Let

$$
R_{12}(u)=\frac{1}{2 i}\left(z R_{12}-z^{-1} R_{21}^{-1}\right)
$$

with $z=e^{i u}$. Then the Yang-Baxter equations $(1.7,1.81)$ for the spectral parameter dependent $R$ matrix $R(u)$ and $R^{\iota_{1}}(u)$ are equivalent to the following equations for the constant $R$-matrices $R$ and $R^{\iota_{1}}$

$$
\begin{aligned}
& R_{12} R_{13}^{\iota_{1}} R_{23}=R_{23}^{\iota_{1}} R_{13} R_{12}^{\iota_{1}} \\
& R_{12}^{\iota_{1}} R_{13} R_{23}^{\iota_{1}}=R_{23} R_{13}^{\iota_{1}} R_{12}
\end{aligned}
$$

$$
\begin{aligned}
& R_{12}\left(R_{31}^{\iota_{1}}\right)^{-1} R_{23}-\left(R_{21}\right)^{-1} R_{13}^{\iota_{1}}\left(R_{32}\right)^{-1}= \\
& R_{23}^{\iota_{1}}\left(R_{31}\right)^{-1} R_{12}^{\iota_{1}}-\left(R_{32}^{\iota_{1}}\right)^{-1} R_{13}\left(R_{21}^{\iota_{1}}\right)^{-1} \\
& R_{12}^{\iota_{1}}\left(R_{31}\right)^{-1} R_{23}^{\iota_{1}}-\left(R_{21}^{\iota_{1}}\right)^{-1} R_{13}\left(R_{32}^{\iota_{1}}\right)^{-1}= \\
& R_{23}\left(R_{31}^{\iota_{1}}\right)^{-1} R_{12}-\left(R_{32}\right)^{-1} R_{13}^{\iota_{1}}\left(R_{21}\right)^{-1}
\end{aligned}
$$

assuming $\tilde{R}=R^{\iota_{1}}$

From the spectral parameter dependent solution we deduce that a solution of $(1.24)$ is given by

$$
\begin{aligned}
R & =\left(\begin{array}{cccc}
q & 0 & 0 & 0 \\
0 & 1 & 0 & 0 \\
0 & q-q^{-1} & 1 & 0 \\
0 & 0 & 0 & q
\end{array}\right), \\
R^{\iota_{1}} & =\left(\begin{array}{cccc}
q & 0 & 0 & 0 \\
0 & -1 & 0 & 0 \\
0 & q-q^{-1} & -1 & 0 \\
0 & 0 & 0 & q
\end{array}\right),
\end{aligned}
$$

where $(4.6)$ is the usual $R$-matrix of $\mathcal{U}_{q}(\operatorname{sl}(2))$.

\section{Algebra}

The $R$-matrices $(\overline{4} \cdot \overline{6})$ and $(\bar{A} \cdot \overline{\overline{7}})$ and the equations (4.2-5. lis lead to the following algebra, defined by the generators $L^{ \pm},\left(L^{ \pm}\right)^{\iota_{1}}$ and the relations

$$
\begin{aligned}
& R_{12} L_{1}^{ \pm \iota_{1}} L_{2}^{ \pm}=L_{2}^{ \pm \iota_{1}} L_{1}^{ \pm} R_{12}^{\iota_{1}} \\
& R_{12} L_{1}^{+\iota_{1}} L_{2}^{-}=L_{2}^{-\iota_{1}} L_{1}^{+} R_{12}^{\iota_{1}} \\
& R_{12}^{\iota_{1}} L_{1}^{ \pm} L_{2}^{ \pm \iota_{1}}=L_{2}^{ \pm} L_{1}^{ \pm \iota_{1}} R_{12} \\
& R_{12}^{\iota_{1}} L_{1}^{+} L_{2}^{-\iota_{1}}=L_{2}^{-} L_{1}^{+\iota_{1}} R_{12}
\end{aligned}
$$

$$
\begin{gathered}
R_{12} L_{1}^{-\iota_{1}} L_{2}^{+}-\left(R_{21}\right)^{-1} L_{1}^{+\iota_{1}} L_{2}^{-}= \\
L_{2}^{+\iota_{1}} L_{1}^{-} R_{12}^{\iota_{1}}-L_{2}^{-\iota_{1}} L_{1}^{+}\left(R_{21}^{\iota_{1}}\right)^{-1}(5.5) \\
R_{12}^{\iota_{1}} L_{1}^{-} L_{2}^{+\iota_{1}}-\left(R_{21}^{\iota_{1}}\right)^{-1} L_{1}^{+} L_{2}^{-\iota_{1}}= \\
L_{2}^{+} L_{1}^{-\iota_{1}} R_{12}-L_{2}^{-} L_{1}^{+\iota_{1}}\left(R_{21}\right)^{-1}(5.6)
\end{gathered}
$$

Writing the operators $L^{ \pm}$in the form

$$
L^{+}=\left(\begin{array}{cc}
K_{+1} & 0 \\
E & K_{+2}
\end{array}\right), \quad L^{-}=\left(\begin{array}{cc}
K_{-1} & F \\
0 & K_{-2}
\end{array}\right) \text {, }
$$

and similarly for $L^{ \pm \iota_{1}}$, we get the relations

$$
\begin{aligned}
& K_{+1}^{\iota_{1}} K_{-1}=K_{-1}^{\iota_{1}} K_{+1} \\
& K_{+2}^{\iota_{1}} K_{-2}=K_{-2}^{\iota_{1}} K_{+2} \\
& K_{+1}^{\iota_{1}} K_{+2}=-K_{+2}^{\iota_{1}} K_{+1} \\
& K_{-1}^{\iota_{1}} K_{-2}=-K_{-2}^{\iota_{1}} K_{-1} \\
& K_{+1}^{\iota_{1}} K_{-2}=-K_{-2}^{\iota_{1}} K_{+1} \\
& K_{+2}^{\iota_{1}} K_{-1}=-K_{-1}^{\iota_{1}} K_{+2} \\
& K_{+1}^{\iota_{1}} E=q E^{\iota_{1}} K_{+1} \\
& K_{-1}^{\iota_{1}} E=q^{-1} E^{\iota_{1}} K_{-1} \\
& K_{+2}^{\iota_{1}} E=-q^{-1} E^{\iota_{1}} K_{+2} \\
& K_{-2}^{\iota_{1}} E=-q E^{\iota_{1}} K_{-2} \\
& K_{+1}^{\iota_{1}} F=-q^{-1} F^{\iota_{1}} K_{+1} \\
& K_{-1}^{\iota_{1}} F=-q F^{\iota_{1}} K_{-1} \\
& K_{+2}^{\iota_{1}} F=q F^{\iota_{1}} K_{+2} \\
& K_{-2}^{\iota_{1}} F=q^{-1} F^{\iota_{1}} K_{-2} \\
& E^{\iota_{1}} F+F^{\iota_{1}} E= \\
& \left(q-q^{-1}\right)\left(K_{-1}^{\iota_{1}} K_{+2}-K_{+1}^{\iota_{1}} K_{-2}\right) .
\end{aligned}
$$

These equations look like those defining $\mathcal{U}_{q}(\operatorname{sl}(2))$, althought important sign differences appear, in particular in the exchange relations of the generators $K$ 


\section{6. $\mathcal{U}_{q}(g l(2))$ interpretation}

We define the following composite operators:

$$
\begin{aligned}
e & =K_{1}^{\iota_{1}} E \\
f & =K_{2}^{\iota_{1}} F \\
k_{1} & =K_{1}^{\iota_{1}} K_{1} \\
k_{2} & =K_{2}^{\iota_{1}} K_{2} \\
l_{1} & =K_{1}^{\iota_{1}} K_{-1} \\
l_{2} & =K_{2}^{\iota_{1}} K_{-2}
\end{aligned}
$$

These operators satisfy the relations

$$
\begin{aligned}
k_{1} e & =-q^{2} e k_{1} \\
k_{1} f & =-q^{-2} f k_{1} \\
k_{2} e & =-q^{2} e k_{1} \\
k_{2} f & =-q^{-2} f k_{1} \\
{[e, f] } & =\left(q^{2}-1\right)\left(k_{1} l_{2}-k_{2} l_{1}\right) \\
l_{i} e & =-e l_{i} \\
l_{i} f & =-f l_{i}
\end{aligned}
$$

These relations are those of $\mathcal{U}_{-q^{2}}(s l(2))$ with supplementary generators $l_{1}, l_{2}$ anticommuting with $e$ and $f$. The operators

$$
\begin{aligned}
l_{1}^{2}, & l_{2}^{2}, \\
k_{1} k_{2}, & l_{1} l_{2}, \\
C_{2}= & f e+q^{2} k_{1} l_{2}+k_{2} l_{1}
\end{aligned}
$$

are Casimir operators of this algebra.

\section{Perspectives and questions}

Generalisations of this construction to other models is under investigation. The case of the $t-J$

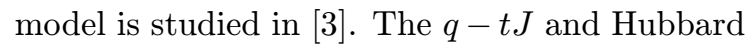
models will also be used as building blocks.

Increasing the number of legs of the ladder would bring us closer to 2-dimensional models.

J.-M. Maillet suggested that the double row monodromy matrices could be permuted using a "bigger" $R$-matrix (instead of permuting single row ones using $R$ and repeating the operation). The answer is that this can be done; more precisely, if $\mathcal{R}$ is the block

$$
\begin{gathered}
\mathcal{R}_{\left\{i_{1} i_{2}\right\}\left\{j_{1} j_{2}\right\}}\left(u, u^{\prime}, v, v^{\prime}\right)= \\
R_{i_{1} j_{1}}^{\iota_{1}}\left(u^{\prime}-v\right) R_{i_{1} j_{2}}\left(u^{\prime}-v^{\prime}\right) \\
R_{i_{2} j_{1}}(u-v) R_{i_{2} j_{2}}^{\iota_{1}}\left(u-v^{\prime}\right)
\end{gathered}
$$

it satisfies the Yang-Baxter equation

$$
\begin{gathered}
\mathcal{R}_{\left\{i_{1} i_{2}\right\}\left\{j_{1} j_{2}\right\}}\left(u, u^{\prime}, v, v^{\prime}\right) \\
\mathcal{R}_{\left\{i_{1} i_{2}\right\}\left\{k_{1} k_{2}\right\}}\left(u, u^{\prime}, w, w^{\prime}\right) \\
\mathcal{R}_{\left\{j_{1} j_{2}\right\}\left\{k_{1} k_{2}\right\}}\left(v, v^{\prime}, w, w^{\prime}\right) \\
=\mathcal{R}_{\left\{j_{1} j_{2}\right\}\left\{k_{1} k_{2}\right\}}\left(v, v^{\prime}, w, w^{\prime}\right) \\
\mathcal{R}_{\left\{i_{1} i_{2}\right\}\left\{k_{1} k_{2}\right\}}\left(u, u^{\prime}, w, w^{\prime}\right) \\
\mathcal{R}_{\left\{i_{1} i_{2}\right\}\left\{j_{1} j_{2}\right\}}\left(u, u^{\prime}, v, v^{\prime}\right)
\end{gathered}
$$

(The indices indicate, as in the YB equations, the spaces in which the matrices act; they are not basis indices.) Moreover, the $2 \times 2$ block of $R$-matrices that is periodically used to construct the double row monodromy matrix is

$$
\mathcal{R}_{\left\{a a^{\prime}\right\}\{2 j-1,2 j\}}(u, \theta-u, 0, \theta) .
$$

(Note that the $\iota_{2}$ transmuted matrices are expressed, using our particular solution (11.94i.1 of the double Yang-Baxter equation $(1.7,1.8)$, as $\iota_{1}$ transmuted ones with opposite spectral parameter.)

\section{Acknowledgments}

This work was partially supported by European Community contract TMR FMRX-CT96.0012. I thank Jean-Michel Maillet for his questions and comments. Finally, many thanks again to the organizers of this lively and interesting conference.

\section{References}

[1] D. Arnaudon, R. Poghossian, A. Sedrakyan, P. Sorba, Integrable Chain Model with Additional Staggered Model Parameter, To appear in Nucl. Phys. B, [hep-th/0002123].

[2] A. De Martino, in these Proceedings.

[3] J. Ambjorn, D. Arnaudon, A. Sedrakyan, T. Sedrakyan, and P. Sorba, Integrable ladder $t-J$ model with staggered shift of the spectral parameter, [hep-th/0006243]. 